\title{
Prevalence of Substance Use Disorders and Effectiveness of Educational Package on Knowledge Regarding the Disorders among Adolescents in Selected Schools of Lekhnath, Nepal
}

\author{
Deepa Gurung ${ }^{1}$, Bindu Thapa ${ }^{2}$ and Amrita Paudel ${ }^{3}$ \\ Lecturer \\ College of Nursing Sciences, Gandaki Medical College, Pokhara, Nepal
}

\begin{abstract}
$>$ Background:

Substance use disorders refers to the abuse of alcohol, illicit drugs, or substances such as over-thecounter medicines, medicines from unsupervised ordinary retail purchase, or even through prescription. It causes significant health problems and functional impairments such as disability and failure to meet responsibilities at work, school, or home. Common substance use disorders are use of alcohol, tobacco, cannabis (marijuana), stimulants, hallucinogens, sedatives, hypnotics, opioids, etc.
\end{abstract}

\section{$>$ Objective:}

To assess prevalence of substance use disorders and effectiveness of educational package on spreading knowledge about substance use disorders among adolescents in selected schools in Lekhnath, Nepal.

\section{> Methodology:}

Pre experimental one group pretest and posttest design was used for the study. Non-probability convenience sampling technique was adopted for sampling. For the study, total of 155 adolescents studying in classes 9 and 10 were selected from schools in Lekhnath, Nepal. Self administered structured questionnaire was used to collect data. Data obtained were entered in Microsoft Excel and analyzed using statistical tool SPSS 16.0.

\section{$>$ Result:}

Among 155 adolescents, 97.40\% had heard of substance use disorders. $62.24 \%$ of people received the information about substance use disorders via mass media. Family members of $16.8 \%$ of samples were engaged in some kind of substance use disorders. $1.90 \%$ of sample students drank alcohol, who all happened to be male of age 15-16 years age group. In pretest, more than half $(69.70 \%)$ of the adolescents had inadequate knowledge about substance use disorders. On the other hand, in the posttest, more than one third of adolescents $(37.4 \%)$ had adequate knowledge and less than twothird adolescents $(\mathbf{5 8 . 1 \%})$ had moderate knowledge on substance use disorders. The paired ' $t$ ' test was found to be significant on knowledge level $(t=23.76$ at $1 \%$
level).Only caste/ethnicity of demographical variable was found to be associated with pretest knowledge regarding substance use disorders $(F=3.495$ at $5 \%$

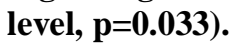

\section{$>$ Conclusion:}

The study concluded that adolescents are in great risk for substance use disorders. After educating on substance use disorders, adolescents gained adequate knowledge regarding the disorder. Study supports that educational package was effective in improving the level of knowledge regarding substance use disorders among adolescents.

Keywords:- prevalence, knowledge, educational package on substance use disorders, adolescents.

\section{INTRODUCTION}

Drug is defined as any substance that when ingested into the living organism may modify one or more of its functions. Drug/substance abuse is a social, economic, psychological and physiological problem worldwide. It is a problem of individual, family and community. It can negatively impact health, relationship, finance, school and employment. ${ }^{1}$ Substance use disorder is a maladaptive pattern of substance use leading to clinically significant impairment or distress; it could also cause social or interpersonal problems, and be physically hazardous (i.e. driving an automobile or operating machinery when impaired by substance use), and could cause legal problems (i.e. arrests for substance-related disorderly conduct). ${ }^{2}$

According to the Undersecretary General of the United Nations, hippie culture in the early 1960's popularized the use of drugs as recreational activity among youth, especially age between 15-30 years old. ${ }^{3}$ The attitude of an individual (whether one laughing or crying, alert or drowsing, happy or sad) depends on specific biochemical activity in the nervous system. Sometimes one attempts to alter that bio chemistry in order to achieve a state of mood or mind which enable him/her to enjoy peace and tranquility through the use of drug. Although one may attempt to alter biochemistry through meditation, relaxation exercise, acupuncture and the like the most common way adopted by some of us is by taking a drug. ${ }^{4}$ 
Substances that are consistently abused among college students are alcohol, Adderall, marijuana, and ecstacy. ${ }^{5}$ In 2010, about $5 \%$ of people (230 million) used illicit substances. Out of those 230 million students, 27 million have high-risk drug use otherwise known as recurrent drug use causing harm to their health, psychological problems, or social problems that put them at risk of those dangers. In 2015, substance use disorders resulted in 307,400 deaths, up from 165,000 deaths in 1990. Of the total death in 2015, alcohol use disorders was responsible for 137,500 deaths, 122,100 death was caused by opioid use disorder, amphetamine use disorder caused 12,200 deaths, and cocaine use disorders was responsible for 11,100 deaths. $^{2}$

Varieties of factors contribute to the development of substance related disorders. ${ }^{6}$ Some biological disorders include genetic vulnerability, comorbid psychiatric disorders, twins study and biochemical factors. Social and environmental disorders are peer pressure, modeling, laws, cost, availability, family conflict, poor parents' support, permissive social attitude and urbanization. Psychological factors are curiosity, poor impulse control, poor stress management skill, childhood loss and boredom. ${ }^{7}$

Substance use disorder can have a wide range of short-term and long-term effects as well as direct and indirect effects which can lead to mental issues including paranoia, depression, anxiety, hallucination and physiological complaints like heart attack, change in blood pressure, lung disease, gastrointestinal problems and neurological effects. ${ }^{8}$ The effects of substance abuse frequently extend beyond the nuclear family. Extended family members may experience feelings of abandonment, anxiety, embarrassment or guilt; they may wish to ignore or cut ties with the person abusing substances. Some family members may feel the need for legal protection from the person abusing substances. ${ }^{9}$

\section{$>$ Rationale of the Study:}

There are 4 million registered drug addicts in South Asia. The distribution is Alcohol 42\%, Heroin 13\%, Cannabis $6.2 \%$, others $1.8 \%$. The majority of drug addicts are aged between 16 to 30 years. These drugs abusers mostly unmarried and lower socio economic group. ${ }^{10}$

Researchers found that with time there is increased in number of adolescents involved in substance use disorders. Educational programs regarding substance use disorders were found to be very effective in creating awareness among adolescents. So far only few studies have been conducted regarding this type of study in Lekhnath. Thus the study was conducted among adolescents in lekhnath, Nepal.

\section{$>$ Objectives:}

- To assess the prevalence of substance use disorders among adolescents.

- To assess the pretest and posttest knowledge of subjects on substance use disorders.

- To evaluate the effectiveness of educational package on knowledge level regarding substance use disorders among adolescents in selected schools of Lekhnath, Nepal.

- To associate pretest knowledge level with their demographical variables.

\section{METHODOLOGY}

According to Health Learning Material Centre (HLMC), Nepal, adolescence is a period of human age from 12 to 18 years. ${ }^{11}$ Two schools in Lekhnath were randomly selected for the study. Pretest and posttest design was used. The samples were selected by non-probability convenience sampling technique. The samples consisted of 155 adolescents studying in classes 9 and 10 of two selected schools at Lekhnath, Nepal. Out of 167 adolescents present in pretest, 12 adolescents were dropped out during the posttest. Thus, the sample size was 155 .

A self-administered structured questionnaire tool was used to assess the knowledge level of the subjects. The tool was tested before the study.

The study was conducted in below three phases:

$>$ Phase I: It includes assessment of the demographical variables, prevalence and pretest knowledge level regarding substance use disorders among adolescents.

$>$ Phase II: On the same day of pretest, 45 minutes lecture was given regarding substance use disorders via multimedia projector.

> Phase III: After 2 days, posttest was conducted.

Ethical clearance was obtained from Institutional Ethical Committee, GMC. Approval for the study was taken from schools and informed consents were taken from each student. The data were collected from December 19, 2018 till December 23, 2019. Finally, data were entered in Microsoft Excel and statistically analyzed by SPSS 16.0. The data obtained were analyzed by using descriptive statistics (like percentage distribution shown in bar, pie charts etc.) and inferential statistics (like paired ' $t$ ' test and ANOVA test). 


\section{RESULTS}

In present study, data were collected from 155 adolescents studying in classes 9 and 10 of selected schools in Lekhnath. Data collected were organized, entered in Microsoft Excel and analyzed and interpreted by descriptive and inferential statistics using SPSS 16.0.

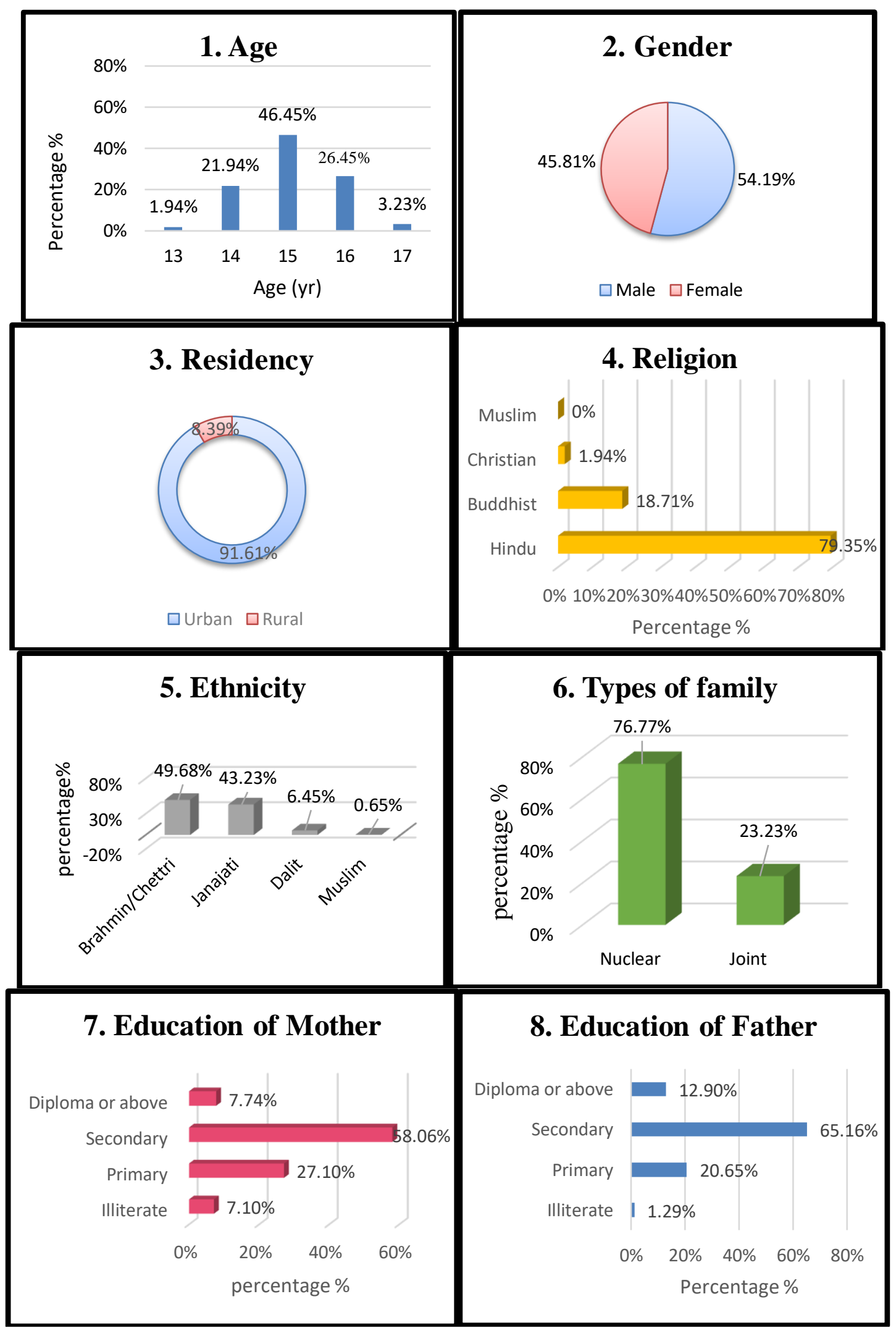

Fig 1:- Percentage distribution of adolescents according to some socio-demographic variables $(n=155)$ 
Figure1: Among 155 adolescents, majority were 15 years old (46.45\%). There were $8.3 \%$ more male than female. Most of the samples were from nuclear family $(76.77 \%)$. Samples with Hindu religion comprised 79.35\%. In Ethnicity, Brahmin/Chettri $(49.68 \%)$ and Janajati (43.23\%) were greater in number. Most of the adolescents lived in urban area (91.61\%). 58.6\% of students had mother with secondary level education, and $65.1 \%$ had father with secondary level education.

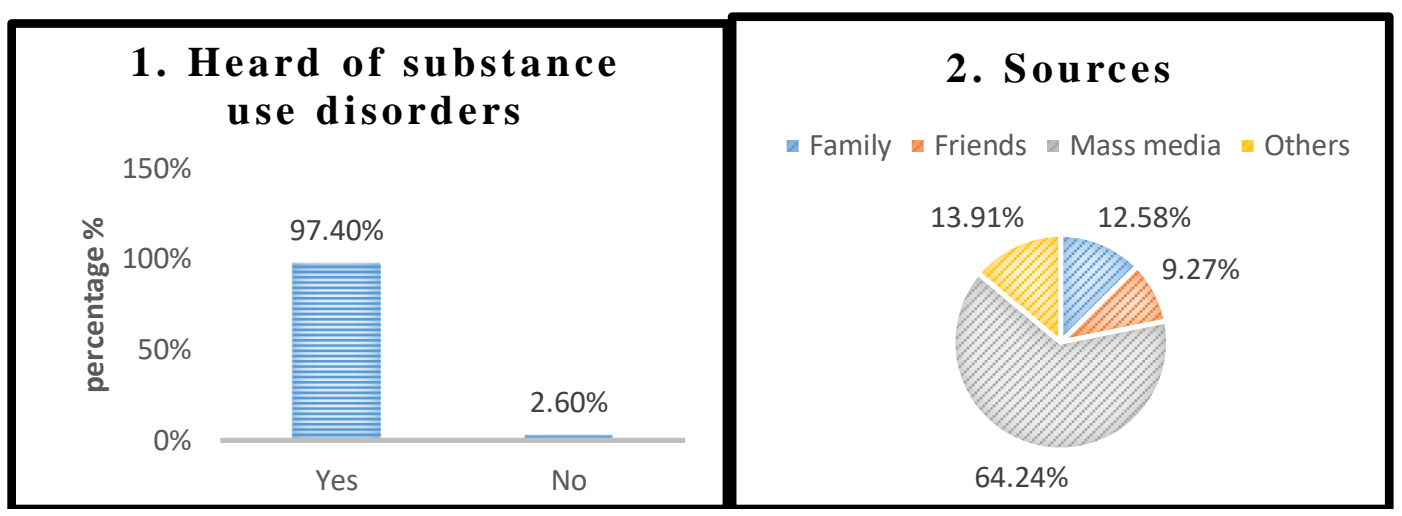

Fig 2:- Percentage distribution of adolescents regarding their source of informationabout the substance use disorders (n=155)

Figure 2: Among 155 adolescents, $97.40 \%$ had heard of substance use disorders from family, friends, mass media and other. Mass media (62.24\%) was found to be the main source of information regarding substance use disorders.

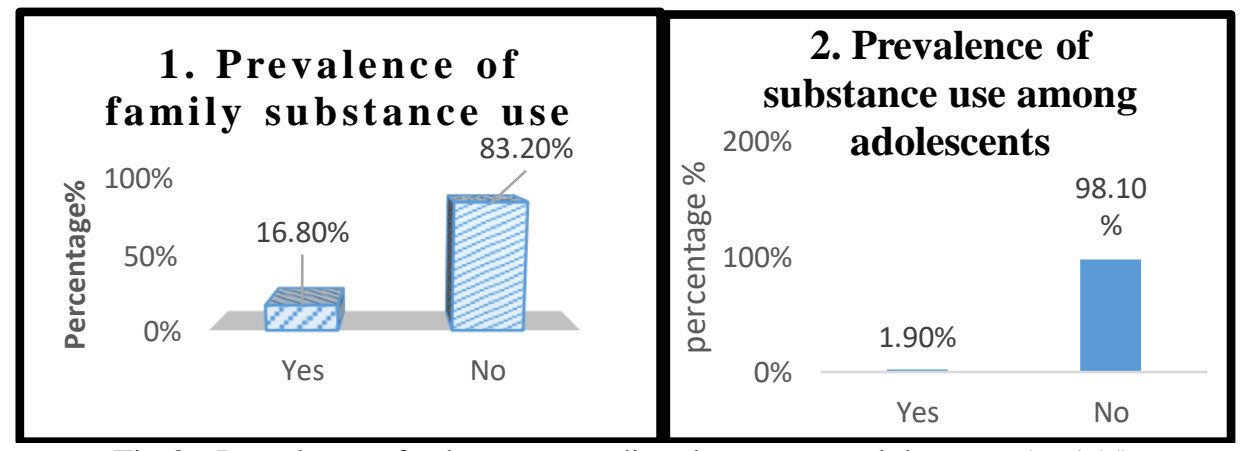

Fig 3:- Prevalence of substance use disorders among adolescents $(\mathrm{n}=155)$

Figure 3: Out of 155 adolescents, family members of $16.8 \%$ used different substances and $1.9 \%$ adolescents were found to be drinking alcohol, who all happened to be made. In that, male of age 15 years $(0.6 \%)$ and 16 years $(1.3 \%)$ were involved. The substances were found to be introduced by family (1.3\%) and remaining $0.6 \%$ got it from friends, suppliers and others.

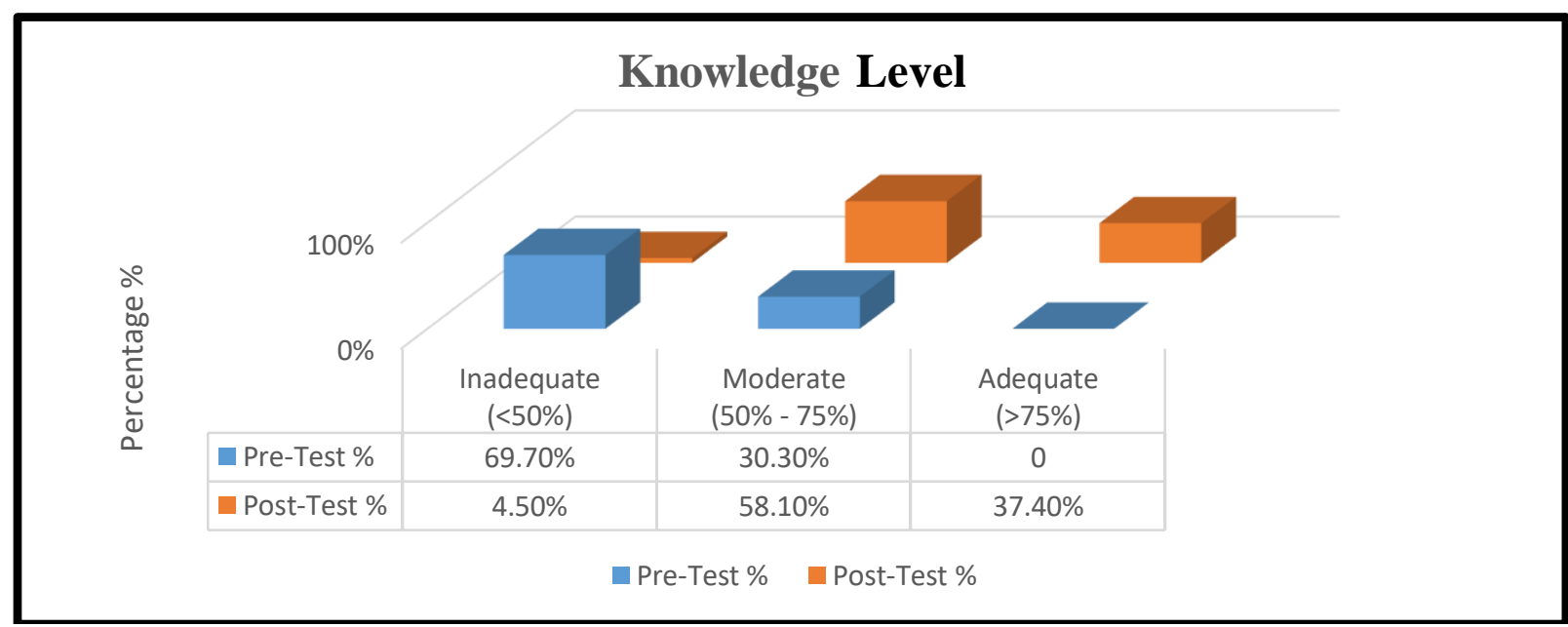

Fig 4:- Percentage distribution of knowledge level of adolescents regarding substance use disorder $(\mathrm{n}=155)$

Figure 4: In pretest, more than half $(69.70 \%)$ of the adolescents had inadequate knowledge and remaining had only moderate knowledge regarding substance use disorders. However, in the posttest, more than one third of adolescents $(37.4 \%)$ had adequate knowledge, little less than two third adolescents $(58.1 \%)$ had moderate knowledge and only remaining few (4.5\%) still had inadequate knowledge of substance use disorder. 
ISSN No:-2456-2165

\begin{tabular}{|c|c|c|c|c|c|c|}
\hline Measures & Pre-Test & Post-Test & Difference & t-value & df & Sig. (2-tailed) \\
\hline Mean & 40.52 & 71.25 & 30.73 & \multirow{2}{*}{$23.76^{*}$} & \multirow{2}{*}{154} & 0.000 \\
\cline { 1 - 3 } Standard Deviation & 10.62 & 12.77 & 16.10 & & \\
\hline
\end{tabular}

Table 1:- Effectiveness of educational package on level of knowledge regarding substance use disorder among adolescents in selected school, Lekhnath, Nepal $(n=155)$

Note: *- denotes significance at $1 \%$ level

In table 1 , the paired ' $t$ ' test was found to be significant on knowledge level ( $\mathrm{t}=23.76$ at $1 \%$ level). It evidence that educational package regarding substance use disorders was found to be effective on knowledge level among adolescents in selected school of Lekhnath.

\begin{tabular}{|c|c|c|c|}
\hline SN & Socio-demographic variables & F-value & P-value \\
\hline 1 & \begin{tabular}{lr}
\multicolumn{2}{l}{ Age (years) } \\
a. & 13 \\
b. & 14 \\
c. & 15 \\
d. & 16 \\
e. & 17 \\
\end{tabular} & 1.648 & 0.165 \\
\hline 2 & \begin{tabular}{cr}
\multicolumn{2}{c}{ Gender } \\
a. & Male \\
b. & female
\end{tabular} & 0.004 & 0.951 \\
\hline 3 & \begin{tabular}{lr}
\multicolumn{2}{c}{ Residence } \\
a. & Urban \\
b. & Rural
\end{tabular} & 0.152 & 0.697 \\
\hline 4 & \begin{tabular}{cc}
\multicolumn{2}{c}{ Religion } \\
a. & Hindu \\
b. & Buddhist \\
c. & Christian \\
d. & Muslim \\
e. & Other \\
\end{tabular} & 1.895 & 0.154 \\
\hline 5 & \begin{tabular}{ccc} 
& \multicolumn{2}{c}{ Ethnicity } \\
& a. & Dalit \\
& b. & Janjati \\
& c. & Muslim \\
d. & & Brahmin/Chettri
\end{tabular} & 3.495* & $\mathbf{0 . 0 3 3}$ \\
\hline 6 & \begin{tabular}{l}
\multicolumn{2}{c}{ Type of family } \\
a. Joint \\
b. $\quad$ Nuclear
\end{tabular} & 2.331 & 0.129 \\
\hline 7 & 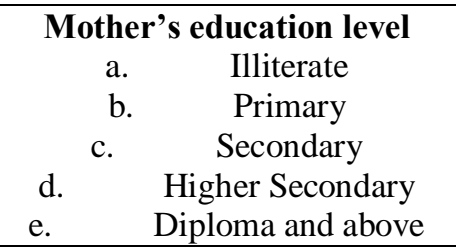 & 2.009 & 0.115 \\
\hline 8 & 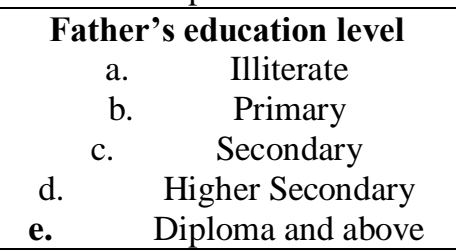 & 1.233 & 0.300 \\
\hline
\end{tabular}

Table 2:- Association of pretest knowledge on substance use disorders with their demographical variables $(\mathrm{n}=155)$

Note: *-denotes significance at $5 \%$ level

Table 2: In ANOVA test values, caste/ethnicity of demographical variable was found to be associated with pretest knowledge on substance use disorders $(\mathrm{F}=3.495$ at $5 \%$ level, $\mathrm{p}=0.033)$. 


\section{DISCUSSION}

The study shows that among 155 adolescents, $97.40 \%$ had heard of substance use disorders from family, friends, mass media and other sources. Mass media (62.24\%) was found to be the main source of information regarding substance use disorders. $16.8 \%$ family members was found to be abusing different substances. Among all, $1.9 \%$ of adolescents were found to be drinking alcohol; they all happened to be male. Among those 1.9\% alcohol users, $0.6 \%$ was 15 years, and $1.3 \%$ was 16 years old. Similarly, $1.3 \%$ claimed to be introduced to alcohol by family members, and $0.6 \%$ mentioned that their friends, and other people introduced them to alcohol. The finding of the study was supported by WHO, alcohol consumption worldwide about $16.0 \%$ of drinkers aged 15 years or older engage in heavy episodic drinking. ${ }^{12}$ The increase coincides with changes in laws and business that enabled a high volume of cheap alcohol to flow into stores and homes. In addition, as much as 40 million liters of alcohol per year, both beers (called Jand or Chyang) and distilled spirits (Raksi), are manufactured in homes. ${ }^{13}$ Television, radio, film, and popular music are often identified as potential sources through which young people learn about alcohol and as potential influences on young people's drinking and drinking problems. ${ }^{14}$

Similarly, a population-based cross-sectional study was conducted by Jenny John Cheriathuto to assess the knowledge of effects of drinking alcohol and attitude towards anti-substance use campaign among school students in Ajman, grades ix-xii, UAE. 411 students between 15-17 years were studied (male students constituted $55.7 \%$, mean age: $15.65 \pm 1.1$ years). A pretested, close-ended questionnaire relating to knowledge of health and social effects of alcohol and attitude towards anti-substance use campaign was administered to the students. The result showed that 375 (91.2\%) students indicated alcohol consumption was harmful, and 288 $(70.1 \%)$ students were aware of the use alcohol. Severe health-associated risks were attributed to drinking alcohol by $63 \%$. About $334(81.3 \%)$ responded that social problems were associated with alcohol consumption. The study concluded that female students were more aware of the reasons for initiation and health and social problems than males. $^{15}$

In the study, in pretest, more than half $(69.70 \%)$ of the adolescents had inadequate knowledge and others had moderate knowledge regarding substance use disorders. Whereas in the posttest, more than one third of adolescents $(37.4 \%)$ had adequate knowledge, little less than two third adolescents $(58.1 \%)$ had moderate knowledge, and only few adolescents $(4.5 \%)$ still had inadequate knowledge on substance use disorders.

A pretest posttest research design was used to determine the effectiveness of awareness program on knowledge on substance abuse and its consequences among pre university students. Sample consisted of 50 students from Udipi district, Karnataka. Sample was collected by convenient sampling method. The tool used was knowledge questionnaire on substance abuse and its consequences. Demographic Performa was used to collect background information. Results show increasing knowledge of students after awareness program on substance abuse and its consequences. ${ }^{16}$

The finding of the study was supported by the study conducted by Vaibhav Janito to assess the effectiveness of planned teaching program on knowledge regarding ill effects of alcohol consumption. The sampling technique used was non-probability convenient sampling. Data was collected from 60 adolescents from selected higher secondary schools of Vadodara district. Data was analyzed using descriptive and inferential statistics. As per the result of the study, in pretest, adolescents had an average $43.43 \%$ knowledge regarding ill effects of alcohol consumption and mean score was $12.43 \pm 2.645$. In posttest, there was average $65.33 \%$ knowledge regarding ill effect of alcohol consumption and mean score was $19.60 \pm 2.599$. t calculated value of -20.948 which is less than the tabulated value of 2.00 at 0.05 level of significance. The study concluded that there is significant difference between pretest and posttest knowledge score of adolescents. ${ }^{17}$

The paired ' $t$ ' test was found to be significant on knowledge level $(\mathrm{t}=23.76 \mathrm{at} 1 \%$ level). It evidence that educational package regarding substance use disorders was found to be effective on knowledge level among adolescents.

The finding of the study was supported by a quasiexperimental control group design study that was conducted by Kalpana Regmito to assess the effectiveness of school based teaching program on knowledge and attitude regarding prevention of substance abuse among adolescents in selected schools of Mangalore, India. Among 100 adolescents, 50 students each were assigned to experimental and control group. Data was collected using validated structured knowledge questionnaire and Likert attitude scale. The ' $t$ ' value $(t=22.29)$ was computed between mean pretest and posttest knowledge scores among experimental group and control group. Also ' $t$ ' value $(\mathrm{t}=21.27)$ was computed between mean pretest and posttest attitude scores among experimental group and control group .The results revealed that there was a significant increase in knowledge and attitude score among experimental group. ${ }^{18}$

Similarly another quasi experimental, one group pretest post-test design was adopted by Lucy Thomas to assess the effectiveness of role play on knowledge regarding substance abuse among 60 adolescents from grade 8,9, and 10 at Krishna Charitable Trust's English Medium School and Junior College in Karad, Maharashtra, India. The paired ' $\mathrm{t}$ ' test value was $15.363(\mathrm{p}<0.0001)$ showing a significant increase in knowledge regarding substance abuse. The study showed that role play on substance abuse was effective in improving the knowledge of adolescents. ${ }^{19}$ 
$>$ Implications of the Study:

This study is useful for Nepal government drug control division, organizations (NGOs, INGOs) who are working in drug abuse control programme and rehabilitation. Here by following suggestion are made:

- The adolescents should be provided adequate knowledge regarding substance use disorders.

- Continuing educational programs can be conducted among adolescents in schools.

- Workshops, symposium, discussion and demonstration can be arranged in educational institutions regarding substance use disorders.

- The students should be given responsibility to spread awareness among the public regarding substance use disorders.

- Large research studies can be conducted regarding making awareness to public regarding substance use disorders.

- This study will serve as a valuable reference material for future research investigator to extend the study area.

\section{$>$ Limitations}

- The sample size was limited to 155 adolescents.

- Non probability sampling technique was used.

\section{CONCLUSION}

The adolescents are in great risk of substance use disorders. $1.9 \%$ male adolescents were found to be drinking alcohol. In pretest, majority of the adolescents $(69.70 \%)$ had inadequate knowledge regarding the disorders. Whereas, after the education regarding the disorders, more than half of the adolescents $(58.1 \%)$ had moderate knowledge and more than one third adolescents (37.4\%) had adequate knowledge regarding the disorders. The pair ' $t$ ' test was found to be significant on knowledge level $(\mathrm{t}=23.76$ at $1 \%$ level $)$. It concluded that educational package was effective in improving the knowledge level regarding substance use disorders among the adolescents.

\section{ACKNOWLEDGEMENT}

The authors owe gratitude to Professor Prakash Baral, Statistician Krishna Giri, the selected schools and the participants to complete the study.

\section{REFERENCES}

[1]. D Elakkuvana B R. DEBR's Mental Health (Psychiatric) Nursing. Bangalore. EMMESS Medical Publishers; 2014.

[2]. Center for Substance Abuse Treatment. Managing Chronic Pain in Adults With or in Recovery from Substance Use Disorders [e-book]. Rockville (MD): Substance Abuse and Mental Health Services Administration (US); 2012[cited 2019 July 03]. Available from: https://www.ncbi.nlm.nih.gov/books/NBK92048/pdf/B ookshelf_NBK92048.pdf
[3]. Ritcher D. Addiction \& Brain damage. London: London University Park Publishers; 1980.

[4]. Duncar D. Drugs and the whole person. USA: USA Library of Congress Publications; 1990.

[5]. Lawson J, Williams M. College Drug Abuse: College Students \& Drug Abuse. Orlando, Addiction Center. Available from: https://www.addictioncenter.com $>$ college.

[6]. Sharma C, Sharma P. Essentials of Psychiatric \& Mental Health Nursing. $2^{\text {nd }}$ ed. Kathmandu: Saurav \& Awish Publisher; 2016.

[7]. Townsend M C. Psychiatric mental Health Nursing. $8^{\text {th }}$ ed. Philadelphia: F. A. Davis Company; 2015: 365413.

[8]. Volkow N D. Health Consequences of Drug Misuse. North Bethesda, National Institute on Drug Abuse. Available from: https://www.drugsbuse.gov/relatedtopics/health-consequences-drus-misuse.

[9]. Edward Kaufman, Marianne R.M. Yoshioka. Substance Abuse Treatment And Family Therapy: A Treatment Improvement Protocol TIP 39[e-book]. $2^{\text {nd }}$ ed. Rockville: DHHS; 2005[cited 2019 June 25]. Available from: https://www.ncbi.nlm.nih.gov /books/NBK64265/pdf/Bookshelf_NBK64265.pdf

[10]. Sreevani R. A guide to Mental Health and Psychiatric Nursing. New Delhi: Jaypee Brother's medical publications Pvt. Ltd: 2004.

[11]. HLMC. Nursing Care of Children. Kritipur: Tribhuvan University Press; 2006.

[12]. Cibulskis R. Management of substance abuse. Geneva, World Health Organization. Available form: https://www.who.int/substance _abuse/facts/alcohol/en/

[13]. Hadley K, Brabenec R, Birkenshaw S, Good J, Oisan A. Nepal drugs abuse and treatment. Nepal. Hollywood Blvd, Narconon International. Available from: https://www.narconon. org/druginformation/nepal-drug-abuse-treatment.html

[14]. Grube J W.Reducing Underage Drinking: A Collective Responsibility [e-book]. Washington: The National Academies Press; 2004[cited 2019 June 25]. Available from: https://www.ncbi.nlm.nih.gov/books /NBK37586/

[15]. Cheriathu J, John L J, Muttappallymyalil J, Sreedharan J, Sharbatti S A, D'Souza E. Knowledge of effects of drinking alcohol and attitude towards anti-substance use campaign among school students in Ajman, Uae. Gulf Medical Journal. 2012 [cited 2019 June 25]; 1(S2):S139-S143. Available from: http://www.gulfmedicaljournal.com/ download /supplement2_12/sup2-12\%20(30).pdf

[16]. Theou C, Nayak A K, Jose T. A study to determine the effectiveness of an awareness programme on knowledge on substance abuse and its consequences among the students of a selected pre university college of Udupi district, Karnataka. Nitte University Journal of Health Science. 2015 March [cited 2019 June 25]; Vol. 5(1). 53-57. Available from: http://nitte.edu.in/journal/december2014/astd.pdf. 
[17]. Jani V, PGN S, H.N R, Varghese R. Effectiveness of planned teaching programme on knowledge regarding ill effects of alcohol consumption. IOSR Journal of Nursing and Health Science. 2014 [cited 2019 June $25]$ $3(5): 16-21$.

Available

from:http://www.iosrjournals.org/iosrjnhs/papers/vol3-issue5/Version-2/C03521621.pdf.

[18]. Regmi K, Jacob V. An evaluative study to determine the effectiveness of school based teaching programme on knowledge and attitude regarding prevention of substance abuse among adolescents in selected schools of Mangalore. International Journal of Recent Scientific Research. 2015[cited 2019 June 25]; 6 (8):5584-5587. Available from: http://recentscientific.com/sites/default/files/3054.pdf

[19]. Thomas L, Naregal P, Mohite V, Tata S H. Effectiveness of Role Play on Knowledge of Adolescents Regarding Substance Abuse. JKIMSU.2015 [cited 2019 June 25]; 4(2):47-54. Available from: https://www.researchgate.net/publication/282077225_ Effectiveness _of_Role_Play_on_Knowledge_of_Adolescents_Rega rding_Substance_Abuse 